\title{
Генерация тока в структурах Pd/InP в атмосфере водорода
}

\author{
() В.А. Шутаев ${ }^{1}$, Е.А. Гребенщикова ${ }^{1}$, В.Г. Сидоров ${ }^{2}$, Ю.П. Яковлев ${ }^{1}$ \\ ${ }^{1}$ Физико-технический институт им. А.Ф. Иоффе Российской академии наук, \\ 194021 Санкт-Петербург, Россия \\ 2 ООО „АИБИ“, \\ 194021 Санкт-Петербург, Россия \\ E-mail: vadimshutaev@mail.ru
}

Поступила в Редакцию 30 июля 2021 г.

В окончательной редакции 2 августа 2021 г.

Принята к публикации 2 августа 2021 г.

Исследован механизм генерации тока в диодах Шоттки Pd/InP в интервале температур 90-300 K в вакууме и в атмосфере азотно-водородных смесей при концентрациях водорода от 4 до 100 об\%, а также при освещении структуры излучением светодиода с длиной волны 0.9 мкм, соответствующей краю поглощения в InP. Показано, что при низкой температуре $(T=90 \mathrm{~K})$ вольт-амперные характеристики в условиях вакуума имеют выпрямляющий характер с высотой барьера 130-150 мэВ. При подаче азотно-водородной смеси высота барьера с увеличением температуры до $300 \mathrm{~K}$ уменьшается практически до нуля из-за уменьшения работы выхода палладия. Показано, что при одновременном воздействии освещения $(\lambda=0.9$ мкм $)$ и азотноводородной смеси в структуре возникают два противоположно направленных потока электронов, один из которых связан со светодиодным освещением, а другой с абсорбцией водорода в слое палладия.

Ключевые слова: палладий, Pd/InP, водород, работа выхода, потенциальный барьер.

DOI: 10.21883/FTP.2021.12.51712.9725

\section{1. Введение}

Палладий $(\mathrm{Pd})$ обладает уникальной способностью поглощать водород в больших количествах (один объем $\mathrm{Pd}$ может поглотить до тысячи объемов водорода $[1,2])$. Причем палладий поглощает водород из смеси газов избирательно и поэтому используется в различных структурах при создании сенсоров водорода для определения присутствия водорода или измерения его концентрации в окружающей среде $[3,4]$.

Палладий, насыщенный водородом, исследуется давно и достаточно интенсивно. Установлено, что при поглощении водорода изменяются многие свойства палладия: уменьшается работа выхода [5], изменяется структура образуются гидриды палладия [6,7], при этом существенно увеличивается постоянная решетки [8], изменяется электрическое сопротивление и может возникать сверхпроводимость [9-11], изменяются оптические свойства $[12,13]$.

В то же время механизмы взаимодействия водорода с палладием изучены еще явно недостаточно. Предполагается, что молекулы водорода в палладии диссоциируют на атомы [7], которые, возможно, ионизируются с образованием свободных электронов и свободных или связанных протонов [14]. Во всяком случае, теория допускает снижение энергии ионизации водорода в Pd до нуля [15]. Несмотря на то что прямые экспериментальные доказательства ионизации водорода в Pd отсутствуют, при интерпретации свойств структур, включающих слой $\mathrm{Pd}$, предположение об ионизации водорода используется довольно часто $[16,17]$.

Настоящая статья посвящена исследованию механизмов генерации токов в структурах $\mathrm{Pd} / \mathrm{InP}$ при различных температурах в вакууме и в газовой среде с водородом, при электрическом смещении и без него, в темноте и при освещении.

\section{2. Эксперимент}

Исследуемые структуры Pd/InP изготовлены по технологии, описанной в работах $[3,4]$. В качестве подложек при создании структур использованы кристаллы $n$-InP с концентрацией электронов $10^{16} \mathrm{~cm}^{-3}$. Слои палладия толщиной $d_{\mathrm{Pd}}=25$ нм наносились на InP термическим напылением в вакууме. Вольт-амперные характеристики (BAX) структур были измерены в интервале температур 90-300 K в вакууме и в газовых смесях водорода с азотом при концентрациях водорода от 4 до 100 об\% в условиях герметичного криостата, исключающего проникновение внешнего света, и при освещении структур излучением светодиода с длиной волны 0.9 мкм, соответствующего краю поглощения в InP. Генерация токов в структурах при отсутствии внешнего электрического смещения была изучена в полной темноте и при освещении при постоянной подаче азотно-водородных смесей в откачанный до остаточного давления $10^{-3}$ Торр криостат со структурами. При измерениях электрических и фотоэлектрических характеристик структур Pd/InP использовался источник-измеритель KEITLEY-2600A (Keithley Instruments, Inc.) с выводом данных на компьютер.

На рис. 1 представлены вольт-амперные характеристики для типичной из исследованных структур Pd/InP, измеренные в различных условиях: черным цветом показаны BAX, измеренные в темноте в вакууме (кривые 1,3); зеленым цветом - BAX, измеренные в темноте в 


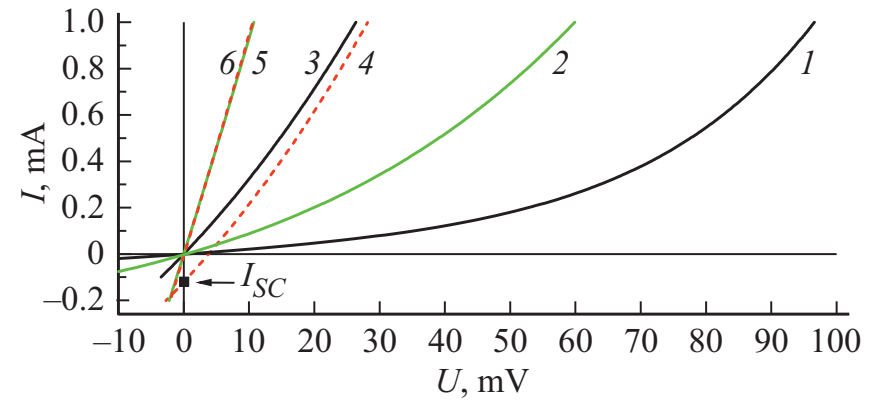

Рис. 1. Вольт-амперные характеристики структуры Pd/InP: 1 и 2 - при 210 К в темноте $(1-$ в вакууме; 2 - в водороде); $3,4,5$ и 6 - при $300 К$ (3 - в вакууме в темноте; 4 в вакууме при освещении излучением светодиода с длиной волны 0.9 мкм при токе через светодиод 50 мА; 5 - в газовой смеси с 10 об\% водорода в темноте; $6-$ в газовой смеси с 10 об\% водорода при освещении излучением светодиода с длиной волны 0.9 мкм при токе через светодиод $50 \mathrm{MA}$ ). (Цветной вариант рисунка представлен в электронной версии статьи).

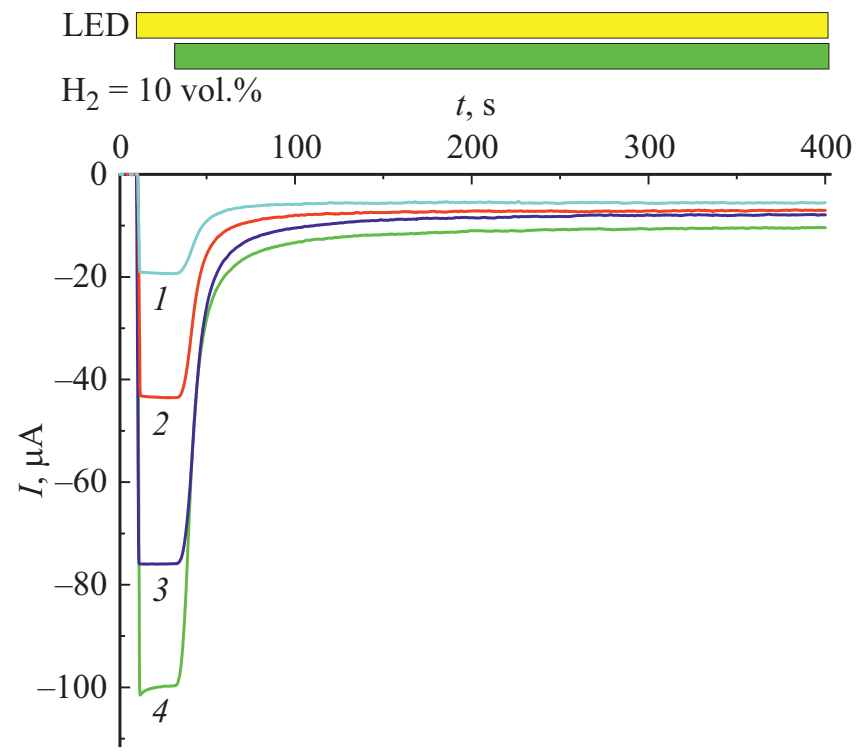

Рис. 2. Кинетика генерации тока при 300 К в структуре Pd/InP в газовой смеси с 10 об\% водорода при различных интенсивностях освещения структуры излучением светодиода с длиной волны 0.9 мкм. Желтое поле указывает на то, что светодиод в этот период времени включен и освещает структуру (ток через светодиод, мА: $1-10,2-20,3-30,4-50)$; зеленое поле соответствует промежутку времени, когда структура находится в среде с водородом.

атмосфере водорода (кривые 2,5); красным цветом $\mathrm{BAX}$, измеренные при освещении структуры излучением от светодиода с длиной волны 0.9 мкм (кривые 4,6); BAX (кривая 4) измерена в вакууме, (кривая 6) измерена в водороде.

На рис. 2 представлена кинетика токов в структуре $\mathrm{Pd} / \mathrm{InP}$ в среде с 10 об\% водорода в темноте, а также при различных освещенностях. После подачи водорода в криостат токи в структуре при разных освещенностях всегда за времена порядка минуты выходят на насыщение и существуют до тех пор, пока в криостате имеется водород, при этом ток в структуре увеличивается пропорционально увеличению интенсивности излучения светодиода. Без водорода эти токи возвращаются к своим прежним значениям.

\section{3. Анализ результатов}

На рис. 3 в увеличенном масштабе показаны особенности характеристик, измеренных в водороде в темноте (кривая 5) и при освещении (кривая 6). Особенность этих характеристик состоит в том, что при увеличении прямого смещения они пересекаются (точка пересечения показана черным квадратом и стрелкой). Это значит, что в структуре существуют два тока, протекающих в противоположных направлениях, а при изменении внешнего напряжения соотношение по величине этих токов изменяется.

На рис. 4 схематически представлены зонные диаграммы структуры Pd/InP, поясняющие измеренные BAX. При анализе будем следить за потоками электронов в структуре. При этом за положительное направление тока электронов примем их перемещение через барьер из InP в $\mathrm{Pd}$ (ток $I_{1}$ на зонных диаграммах, рис. 4), соответствующее прямому электрическому смещению на структуре (положительный потенциал на $\mathrm{Pd}$ ). Ток $I_{2}$ на зонных диаграммах соответствует току неравновесных электронов, возникающих в InP при освещении структуры краевым излучением.

При низкой температуре $(210$ K) BAX и в вакууме, и в водороде (рис. 1, кривые 1 и (2) имеют выпрямляющий характер с высотой барьера $\sim 50$ и $\sim 30$ мэВ соответственно. При 90 К высота барьера увеличивается

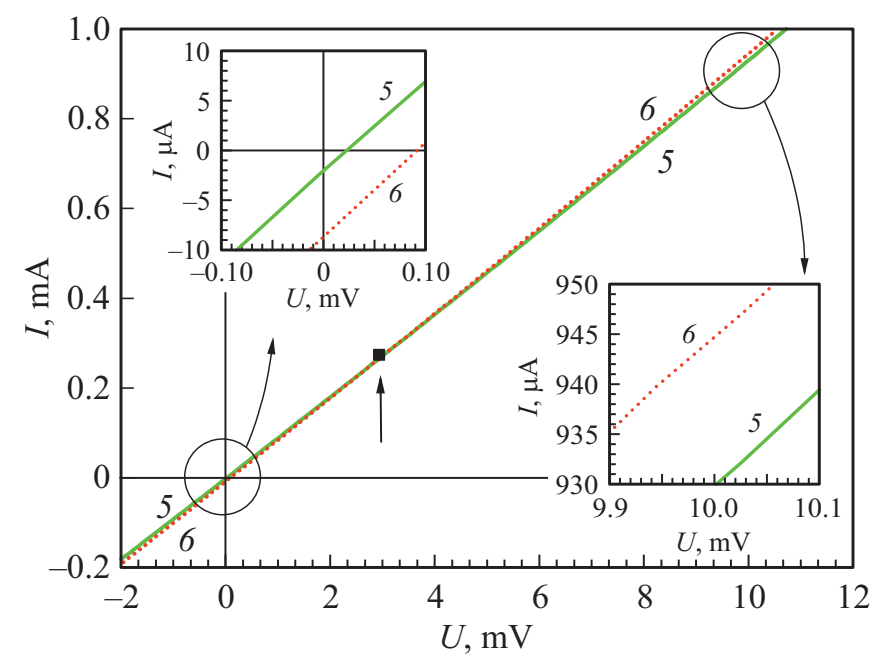

Рис. 3. Вольт-амперные характеристики структуры $\mathrm{Pd} / \mathrm{InP}$, измеренные в газовой смеси с 10 об\% водорода в темноте (5) и при освещении (6); номера и цвет кривых соответствуют обозначениям на рис. 1. 


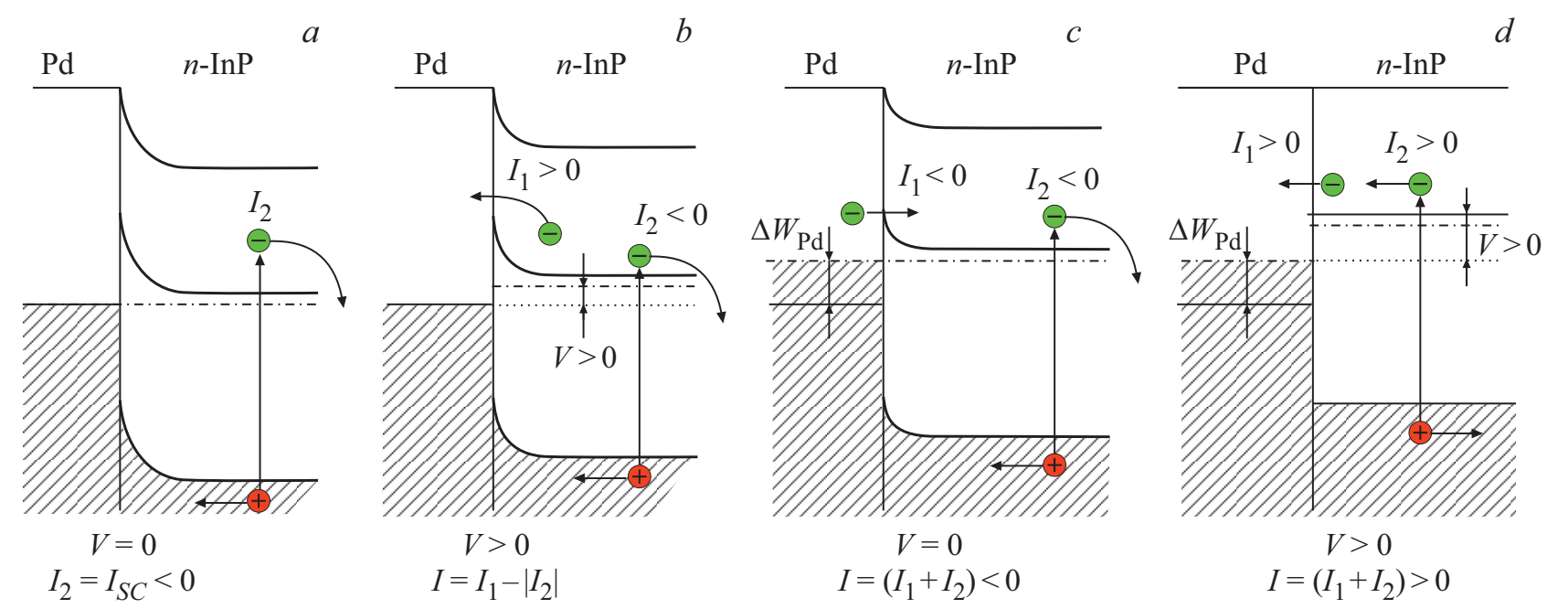

Рис. 4. Схематическое изображение зонных диаграмм и электронных токов в структуре $\mathrm{Pd} / \mathrm{InP}: a$ и $b-$ в вакууме $(a-$ внешнее напряжение на структуре $V=0 ; b-$ внешнее напряжение на структуре $V>0) ; c$ и $d-$ в водороде $(c-V=0 ; d-V>0)$; $I_{1}$ - ток равновесных электронов; $I_{2}$ - ток неравновесных электронов, сгенерированных излучением светодиода.

до 150 и 130 мэВ. При комнатной температуре ВАХ в вакууме в темноте (рис. 1, кривая 3) становится почти линейной. Потенциальный барьер становится существенно прозрачным, но не исчезает совсем, так как при освещении он разделяет генерирующиеся в InP неравновесные носители заряда (рис. $4, a$, ток $I_{2}$ отрицателен и равен току короткого замыкания $I_{S C}$, рис. 1). При прямом смещении ток неравновесных электронов $I_{2}$ направлен противоположно току прямого смещения $I_{1}$ на диоде (рис. 4,b). Полный ток через структуру равен $I=I_{1}-\left|I_{2}\right|$, ВАХ смещается к меньшим токам, а при увеличении прямого смещения ВАХ с освещением (рис. 1, кривая 4) приближается к BAX в темноте (рис. 1, кривая 3), так как часть неравновесных электронов увлекается полем прямого смещения через барьер из $\mathrm{InP}$ в $\mathrm{Pd}$.

В атмосфере водорода работа выхода палладия уменьшается (рис. $4, c$ и $d, \Delta W_{\mathrm{Pd}}$ ), потенциальный барьер на границе Pd и InP почти исчезает, BAX в темноте и при освещении становятся линейными и практически совпадают (рис. 1, кривые 5 и 6). Детальное рассмотрение этих характеристик в увеличенном масштабе (рис. 3) показало, что небольшой барьер на границе раздела всетаки сохраняется, так как при отсутствии смещения на структуре ток неравновесных электронов отрицателен (рис. 2 , кривая 6 , рис. $4, c$, ток $I_{2}$ ). При прямом смещении потенциальный барьер на границе Pd и InP исчезает совсем, а внешнее поле разворачивает электронные токи $I_{1}$ и $I_{2}$ в положительном направлении (рис. $4, d$ ), и $\mathrm{BAX}$ в темноте и при освещении (5 и 6 ) пересекаются. Ток, возникающий при освещении структуры (рис. 3, кривая 6), становится больше темнового тока (рис. 3 , кривая 5).

Следует отметить, что значения наблюдаемых токов в структурах $\mathrm{Pd} / \mathrm{InP}$ количественно мало изменялись в зависимости от концентрации водорода в окружающей среде в пределах от 4 до 100 об\%. Это значит, что палладий, по-видимому, всегда насыщается водородом до вполне определенных пределов, а от концентрации водорода в окружающей среде зависит лишь скорость насыщения. Это также говорит о том, что водород, поглощенный палладием, находится в нем в связанном состоянии. Иначе прослеживалась бы четкая связь между концентрацией водорода в окружающей среде и его концентрацией в палладии. О связанном состоянии водорода в палладии также свидетельствует следующий экспериментальный факт: насыщение палладия водородом происходит за микросекунды-секунды, а освобождение палладия от поглощенного водорода продолжается в течение минут-десятков минут.

Кроме того, резкое изменение свойств $\mathrm{Pd}$ в атмосфере водорода указывает на образование гидрида палладия $\left(\mathrm{PdH}_{x}\right)$, который имеет свойства, отличные от чистого палладия. Однако при десорбции водорода наблюдается восстановление свойств палладиевого слоя [12].

\section{4. Заключение}

Созданы структуры $\mathrm{Pd} / \mathrm{InP}$ и исследованы их вольтамперные характеристики в интервале температур $90-300 \mathrm{~K}$ в вакууме и в атмосфере азотно-водородных смесей при концентрации водорода от 4 до 100 об\%, в темноте и при освещении структуры излучением светодиода с длиной волны 0.9 мкм, соответствующей краю поглощения в InP.

При низкой температуре $(T=90 \mathrm{~K})$ вольт-амперные характеристики структур $\mathrm{Pd} / \mathrm{InP}$ и в вакууме, и в среде с водородом имеют выпрямляющий характер с высотой потенциальных барьеров 150 и 130 мэВ соответственно. С увеличением температуры в среде с водородом высота барьера уменьшается практически до нуля из-за умень- 
шения работы выхода палладия, насыщенного водородом.

Фототок, возникающий в структуре $\mathrm{Pd} / \mathrm{InP}$ при еe освещении излучением с длиной волны края поглощения InP, резко уменьшается в среде с водородом до величины, пропорциональной освещенности структуры. Этот эффект связан с уменьшением высоты потенциального барьера на границе Pd c InP, что в свою очередь вызвано уменьшением работы выхода $\mathrm{Pd}$ в среде с водородом.

Слабая зависимость наблюдаемых токов в структуре $\mathrm{Pd} / \mathrm{InP}$ от концентрации водорода в окружающей среде свидетельствует о том, что насыщение палладия водородом происходит до определенных пределов и что водород в $\mathrm{Pd}$ находится в связанном состоянии.

\section{Конфликт интересов}

Авторы заявляют, что у них нет конфликта интересов.

\section{Список литературы}

[1] А.С. Мокрушин, Р.В. Радченко, В.В. Тюльпа. Водород в энергетике (Екатеринбург, Изд-во Урал. ун-та, 2014) c. 156.

[2] Б.В. Некрасов. Основы общей химии (М., Химия, 1973), T. 2 , c. 382 .

[3] Е.А. Гребенщикова, В.Г. Сидоров, В.А. Шутаев, Ю.П. Яковлев. ФТП, 53 (2), 246 (2019).

[4] В.А. Шутаев, Е.А. Гребенщикова, А.А. Пивоварова, В.Г. Сидоров, Л.К. Власов, Ю.П. Яковлев. ФТП, 53 (10), 1427 (2019).

[5] K. Okuyama, N. Takinami, Y. Chiba, S. Ohshima, S. Kambe. J. Appl. Phys., 76 (1), 231 (1994).

[6] Г.И. Жиров. Физика и техника высоких давлений, 13 (2), $71(2003)$.

[7] C.C. Ndaya, N. Javahiraly, A. Brioude. Sensors, 19 (20), 4478 (2019).

[8] Ю.М. Коротеев, О.В. Гимранова, И.П. Чернов. ФТТ, 53 (5), 842 (2011).

[9] M. Yussouf, B.K. Rao, P. Jena. Solid State Commun., 94 (7), 549 (1995).

[10] P. Hertel. Z. Physic, 268, 111 (1974).

[11] P. Tripodi, D.DI. Gloacchino, J.D. Vinko. Int. J. Mod. Phys. B, 21 (18), 3343 (2007).

[12] В.А. Шутаев, В.Г. Сидоров, Е.А. Гребенщикова, Ю.П. Яковлев. ОПт. и спектр., 128 (5), 603 (2020).

[13] J.I. Avila, R.J. Matelon, R. Trabol, M. Favre, D. Lederman, U.G. Volkmann, A.L. Cabrera. J. Appl. Phys., 107, 023504 (2010).

[14] A. Kawasaki, S. Itoh, K. Shima, T. Yamazaki. Mater. Sci. Eng. A, 551, 231 (2012).

[15] О.В. Константинов, В.Д. Дымников, М.А. Митцев. ФТП, $42(8), 947$ (2008).

[16] K. Skucha, Zh. Fan, K. Jeon, A. Javey, B. Boser. Sensors Actuators B, 145, 232 (2010).

[17] Kh.M. Salikhov, S.V. Slobodchikov, B.V. Russu. SPIE, 3122, 494 (1997).

Редактор А.Н. Смирнов

\section{Current generation in $\mathrm{Pd} / \mathrm{InP}$ structures in hydrogen medium}

\author{
V.A. Shutaev' ${ }^{1}$, E.A. Grebenshchikova ${ }^{1}$, V.G. Sidorov ${ }^{2}$, \\ Yu.P. Yakovlev ${ }^{1}$ \\ ${ }^{1}$ loffe Institute, \\ 194021 St. Petersburg, Russia \\ 2 LLC „Independent Business \& Scientific Group“, \\ 194021 St. Petersburg, Russia
}

\begin{abstract}
The current generation mechanism in $\mathrm{Pd} / \mathrm{InP}$ Schottky diodes has been studied in the range of $90-300 \mathrm{~K}$ in vacuum, hydrogen-nitrogen mixture of concentration from 4 to 100 vol.\%, and under illuminating of the structures by LED with the wavelength of $0.9 \mu \mathrm{m}$, corresponding to the absorption edge in InP. It was shown that at low temperature $(T=90 \mathrm{~K}) I-V$ characteristics of the structures in vacuum have rectifying character with the barrier height of $130-150 \mathrm{meV}$. In hydrogen-nitrogen medium the barrier height decreases almost to zero with increasing the temperature to $300 \mathrm{~K}$ due to palladium work function decreasing. It was demonstrated that under simultaneous impact of illumination $(\lambda=0.9 \mu \mathrm{m})$ and hydrogen-nitrogen mixture there are two opposite directed electron flows in the structures, one of which is related to the LED illumination and the other one to hydrogen absorption in palladium layer.
\end{abstract}

